Received 00th January 20xx, Accepted 00th January 20xx

DOI: $10.1039 / x 0 x x 00000 x$

\title{
Synthesis of PMMA-based block copolymers by consecutive irreversible and reversible addition-fragmentation chain transfer polymerizations
}

\author{
Cédric Bergerbit, $\ddagger^{\mathrm{a}}$ Bárbara Farías-Mancilla, $\ddagger^{\mathrm{b}}$ Lucie Seiler, ${ }^{\mathrm{b}}$ Vincent Monteil, ${ }^{\mathrm{a}}$ Simon Harrisson, ${ }^{\mathrm{b}}$ \\ Franck D'Agosto, *a Mathias Destarac*b
}

\begin{abstract}
Easy to implement reversible-deactivation radical polymerization (RDRP) methodologies for the synthesis of block copolymers from monomers of disparate reactivities are scarce. We report the direct synthesis of PMMA-PVAc block copolymers by addition-fragmentation chain transfer polymerization without resorting to a chemical switch strategy. Instead, we took advantage of the good chain transfer activity of xanthogen and thiuram disulfides in methyl methacrylate (MMA) polymerization in order to obtain xanthate and dithiocarbamate-terminated PMMA (respectively PMMA-X and PMMA-T) displaying terminal functionalities of 0.9. Unlike PMMA-T, the xanthate group of PMMA-X was unstable and tended to partly degrade during the polymer purification. Both types of precursors have been successfully used as macrochain transfer agents for RAFT polymerization of vinyl acetate (VAc). This simple method for block copolymer synthesis can be potentially applied to numerous combinations of more-activated and less-activated monomers. As an example, PMMAT was chain extended with ethylene to achieve, for the first time using a RDRP, block copolymers incorporating PMMA and polyethylene (PE) segments.
\end{abstract}

\section{Introduction}

Reversible-deactivation radical polymerization $(\mathrm{RDRP})^{1}$ techniques are well adapted to the synthesis of copolymers with controlled architectures. ${ }^{2-4}$ Over the years, processes combining more-activated monomers (MAMs, e.g. styrene, (meth)acrylates and (meth)acrylamides) and less-activated monomers (LAMs, e.g. vinyl esters and $\mathrm{N}$-vinyl monomers) for the synthesis of well-defined poly(MAM)-b-poly(LAM) have gained an increasing interest. ${ }^{5-22}$ Some groups have proposed original routes to achieve poly(MAM)- $b$-poly(LAM) by combining different polymerization mechanisms for each block, for example atom transfer radical polymerization (ATRP) ${ }^{6}$ for the poly(MAM) block and reversible addition fragmentation chain transfer $\left(\right.$ RAFT) ${ }^{7}$ polymerization for the poly(LAM) block. ${ }^{8,9}$ The synthesis of such block copolymers by sequential monomer addition using the same polymerization mechanism is far more attractive and RDRPs based on degenerative transfer have shown the most promising results in that respect. Organoheteroatom-mediated living radical polymerization with $\mathrm{Te}, \mathrm{Bi}$ and $\mathrm{Sb}$ derivatives produced numerous MAM-LAM block copolymers, ${ }^{10}$ like well-defined poly(styrene)-b-poly $(N$ vinylpyrrolidone) (PS- $b$-PNVP). ${ }^{11}$ On the other hand, RAFT

\footnotetext{
Université de Lyon, Université Lyon 1, CPE Lyon, CNRS UMR 5265, Laboratoire C2P2, Équipe LCPP, 69616 Villeurbanne CEDEX, France. franck.dagosto@univlyon1.fr

b. Laboratoire des IMRCP, Université Paul Sabatier, CNRS UMR 5623, 118 route de Narbonne 31062 Toulouse, France.destarac@chimie.ups-t/se.fr

Electronic Supplementary Information (ESI) available: See DOI: 10.1039/x0xx00000x ¥ These authors contributed equally

Electronic Supplementary Information (ESI) available: [details of any supplementary information available should be included here]. See DOI: 10.1039/x0xx00000x
}

polymerization has been frequently used and stands out as one of the most robust and versatile processes for the preparation of such block copolymers. Switchable chain transfer agents (CTAs) such as $\mathrm{N}$-methyl- $\mathrm{N}$-(4-pyridinyl) $)^{12-14}$ and $\mathrm{N}$-aryl- $\mathrm{N}$-(4pyridinyl $)^{15}$ dithiocarbamates control either MAMs upon protonation by a strong acid, or LAMs after deprotonation by a base. CTAs with intermediate reactivities, such as 3,5-dimethyl$1 \mathrm{H}$-pyrazole-1-carbodithioates, have also enabled the synthesis of PDMA- $b$-PVAc ${ }^{16}$ without the need for an external stimulus. Alternatively, $\mathrm{O}$-ethyl xanthates, ${ }^{17}$ fluoroxanthates ${ }^{18}$ or $\mathrm{N}, \mathrm{N}$ dithiocarbamates $^{19}$ offer moderate control over MAMs (styrene ${ }^{19}$, acrylates ${ }^{20}$ and acrylamides ${ }^{21}$ ) and good control over LAMs. Very recently, Müllner and coworkers combined a xanthate and a bismuth oxide photocatalyst to provide excellent control over both LAMs and MAMs. PDMA- $b$-PVAc and PDMA- $b$-PNVP copolymers with high molar mass were obtained with remarkably low dispersities. ${ }^{22}$

Among MAMs, methacrylates have several advantages compared to their acrylate counterparts, such as much higher $\mathrm{T}_{\mathrm{g}}$, greater industrial availability and lower susceptibility to hydrolysis. Their use in RAFT polymerization is well established, but the synthesis of poly(methacrylate)-block-poly(LAM) copolymers remains a challenge. For instance, 3,5-dimethyl- $1 \mathrm{H}$ pyrazole-1-carbodithioates are efficient for acrylamides but not for MMA. The control of the polymerization of MMA could be slightly improved by introducing halogen atoms at the 4position on the pyrazole ring, but this negatively affected the polymerization of the LAM. ${ }^{23}$ To the best of our knowledge, there are only three examples of the synthesis of PMMA- $b$-PVAc by RAFT via the use of (i) switchable dithiocarbamates ${ }^{12}$, (ii) fluorinated xanthates in a semi-batch emulsion process, ${ }^{18}$ or (iii) diselenocarbonate (Se-RAFT) agents. ${ }^{24}$ however, PMMA- $b$-PVAc copolymers are potentially attractive to tailor interfaces in 


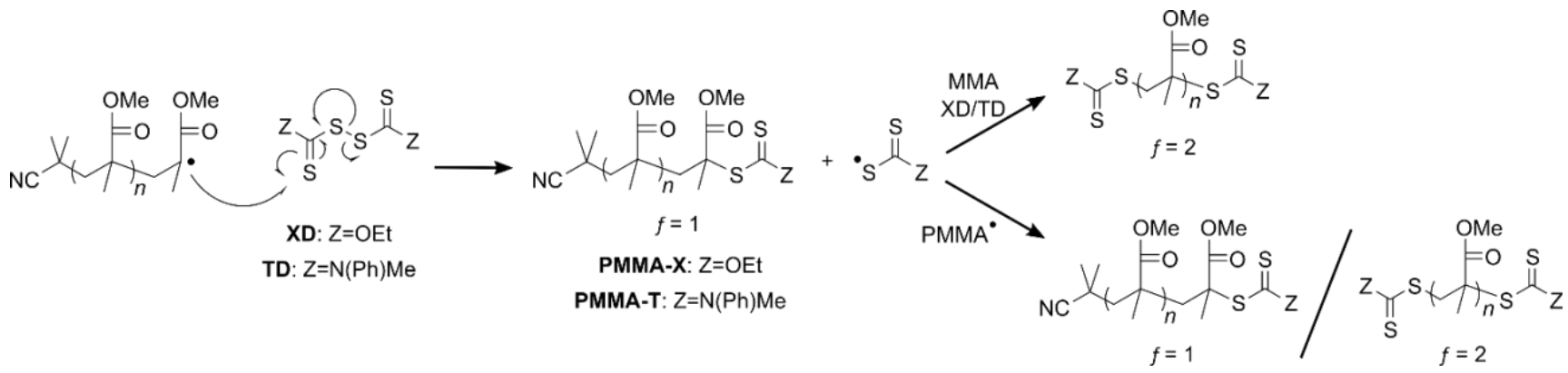

Scheme 1 Mechanism of formation of PMMA-X and PMMA-T using XD and TD respectively and possible formation of monofunctional or telechelic PMMA by either primary termination or reinitiation by a XD/TD radical fragment.

PMMA/PVAc blends ${ }^{25}$ or to generate nanostructured phases in epoxy networks (benefiting from the miscibility of MMA block with epoxy-amine formulations). ${ }^{26}$ In addition, they could be selectively hydrolyzed leading to amphiphilic PMMA-b-PVA copolymers (VA: Vinyl Alcohol).

The limited number of methods to prepare PMMA- $b$-poly(LAM) by RAFT polymerization has led us to explore alternative options. In the late 50's, Otsu et al. introduced the iniferter concept with the use of xanthogen and thiuram disulfides as thermal and photo-polymerization initiators for vinyl monomers. ${ }^{27,28}$ Subsequent developments enabled the synthesis of telechelic (i.e difunctionalized) PMMA by photopolymerization of MMA in the presence of either diisopropyl xanthogen disulfide (xanthate-functionalized PMMA) ${ }^{29}$ or a tetralkyl thiuram disulfide (dithiocarbamatefunctionalized PMMA). ${ }^{30}$ The resulting telechelic PMMAs were used to prepare A-B-A type triblock copolymers with styrene under UV irradiation. ${ }^{31,32}$ On the other hand, the conventional polymerization of MMA (initiated by a thermal radical initiator) in the presence of diethyl xanthogen disulfide ${ }^{33}$ or isopropylxanthic disulfide ${ }^{34}$ has been shown to afford xanthateterminated PMMA (PMMA-X). However, conclusions about xanthate functionality $f$ differ depending on authors. Niwa et al. ${ }^{27}$ determined a $f$ value close to 2 by UV analysis whereas Catala ${ }^{33}$ and Zhang et al. ${ }^{34}$ hypothesized a mechanism dictated by chain transfer to xanthogen disulfide and primary termination of xanthyl radicals, i.e. $f=1$. Some of the resulting PMMAs have been used as macromolecular CTAs (macro-CTAs) and chain-extended in a controlled fashion with a LAM (VAc ${ }^{33}$ and $\mathrm{NVP}^{34}$ ) to afford a PMMA- $b$-poly(LAM) according to a RAFT mechanism, without the need for a switchable RAFT agent. Although very promising, this $\mathrm{MMA} /$ xanthogen disulfide system was limited to low MMA conversions $(<40 \%)^{34}$ and no experimental data for $f$ was given to support the proposed mechanism, which motivated a more detailed study from a kinetic and mechanistic point of view.

In this publication, we determine the end-functionality and elucidate the kinetics and mechanisms of formation of PMMA chains when initiated by AIBN in the presence of diethyl xanthogen disulfide (XD, Scheme 1). We reveal a practical problem related to the instability of the PMMA-xanthate upon prolonged handling. Block copolymer synthesis was facilitated by considering $N, N^{\prime}$-dimethyl- $N, N^{\prime}$-diphenyl thiuram disulfide (TD, Scheme 1) as an alternative irreversible additionfragmentation chain transfer agent for MMA polymerization in a first step, followed by RAFT polymerization of VAc using the resulting PMMA-T as macro-CTA to achieve PMMA- $b$-PVAc. In the last part of the manuscript, we apply recent advances in the RDRP of ethylene using RAFT ${ }^{35,36}$ to produce block copolymers incorporating polyethylene (PE) and PMMA segments by chain extension of PMMA-T with ethylene.

\section{Materials and Methods}

Methyl methacrylate (MMA, Aldrich, $99 \%$ ) and vinyl acetate (VAc, Aldrich, $99 \%$ ) were purified by passing through a basic alumina column. Ethylene (99.95 \%, Air Liquide), 2,2'-azobis(2methylpropionitrile (AIBN, Aldrich, $99 \%$ ) was purified by recrystallization in methanol. Tetrahydrofuran (THF, Fisher, HPLC) was distilled over sodium/benzophenone prior to use. Potassium ethyl xanthogenate (Aldrich, $96 \%$ ), carbon disulfide (Aldrich, anhydrous, $\geq 99 \%$ ), $N$-methylaniline (Aldrich, $98 \%$ ), $n$ butyl lithium (Aldrich, $2.5 \mathrm{M}$ in hexanes), iodine $\left(I_{2}\right.$, Acros Organics, $99 \%$ ), potassium iodide (KI, Acros Organics, $98 \%$ ), toluene (Aldrich, $99 \%$ ), acetonitrile (MeCN, Fisher, HPLC), hexane (Fisher, HPLC), dimethyl carbonate (DMC) and dichloromethane (DCM, Aldrich, ACS reagent) were used as received.

Monomer conversions and chain-end functionality were determined by ${ }^{1} \mathrm{H}$ nuclear magnetic resonance $\left({ }^{1} \mathrm{H} \mathrm{NMR}\right.$, Bruker AM $400 \mathrm{MHz}$ ) spectroscopy. The resonance of the solvent $\left(\mathrm{CDCl}_{3}, \delta=7.26 \mathrm{ppm}\right)$ was used for reference.

Number- $\left(M_{\mathrm{n}}\right)$ and weight-average molar masses $\left(M_{\mathrm{w}}\right)$ and the corresponding dispersities $(\theta)$ were determined by size exclusion chromatography. PMMA and PMMA- $b$-PVAc were analyzed in THF with two Styragel HR3-HR4 columns $(7.8 \mathrm{~mm} x$

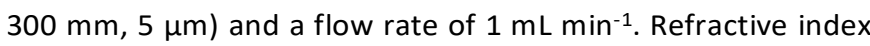
(RI), multi-angle light scattering (MALS) and UV detectors were used. Samples containing a PE block were analyzed in 1,2,4trichlorobenzene at $150{ }^{\circ} \mathrm{C}$ (HT-SEC) using a Viscotek system (Malvern Instruments) equipped with three columns (PLgel Olexis $300 \mathrm{~mm} \times 7 \mathrm{~mm}$ from Agilent Technologies) and a RI detector. Samples were eluted at $1 \mathrm{~mL} \mathrm{~min}-1$ and 2,6-di-tertbutyl-4-methylphenol ( $200 \mathrm{mg} \mathrm{L}^{-1}$ ) was used as stabilizer for the mobile phase.

Matrix-assisted laser desorption and ionization-time-of-flight mass spectrometry (MALDI-TOF MS) measurements were performed on an applied Biosystems Voyager System 4243. The polymer sample and the dithranol matrix were dissolved in THF and premixed in a 1:3 volume ratio. 


\section{Experimental procedure}

Preparation of XD. Diethyl xanthogen disulfide (XD) was prepared as described in the literature. ${ }^{37}{ }^{1} \mathrm{H}$ NMR $\left(\mathrm{CDCl}_{3}\right) \delta$ (ppm) 4.70 (q, 4H, 2x CH $\mathrm{CH}_{2}$; $1.43\left(\mathrm{t}, 6 \mathrm{H}, 2 \mathrm{x} \mathrm{CH}_{3}\right) .{ }^{1} \mathrm{H} \mathrm{NMR}$ spectrum is shown in Fig. $\mathrm{S} 1$.

Preparation of TD. To a cold $\left(-20^{\circ} \mathrm{C}\right)$ solution of $\mathrm{N}$-methylaniline $(12 \mathrm{~g}-0.111 \mathrm{~mol})$ in dry THF $(50 \mathrm{~mL})$ was added $n$-butyl lithium (47 mL $-0.117 \mathrm{~mol}$ ) over 20 minutes under Argon. A white precipitate appeared upon addition. The suspension was allowed to stir for 1 hour at that temperature and cooled down to $-20{ }^{\circ} \mathrm{C}$ before the dropwise addition of $\mathrm{CS}_{2}(10 \mathrm{~mL}-0.167$ $\mathrm{mol})$. The resultant bright orange solution was allowed to stir at room temperature overnight. The reaction mixture was cooled to $0^{\circ} \mathrm{C}$ and an iodine solution $\left(14 \mathrm{~g} \mathrm{I}_{2}\right.$ dissolved in $250 \mathrm{~mL} 10 \%$ aqueous $\mathrm{KI}$ solution) was added dropwise. More water was added and THF was removed in vacuo to afford a thick suspension. The solid was isolated by filtration, washed with water, methanol and heptane until colorless filtrate. The crude product was recrystallized from DCM/MeOH to afford TD as a tan colored crystalline powder that was dried in vacuo until stable weight ( $15.71 \mathrm{~g}, 78 \%$ yield). ${ }^{1} \mathrm{H}$ NMR $\left(\mathrm{CDCl}_{3}\right) \delta(\mathrm{ppm}) 3.82$ (s, 6H, 2x N-CH $) ; 7.45(\mathrm{~m}, 10 \mathrm{H}, 2 \times \operatorname{ArH})$. LC-MS $(\mathrm{M}+\mathrm{H})^{+} \mathrm{m} / \mathrm{z}=$ 365.0269 (365.0267 theo.). ${ }^{1} \mathrm{H}$ NMR spectrum is shown in Fig. S2.

Determination of chain transfer constant by Mayo plot. A fixed quantity of MMA and AIBN were introduced in a Schlenk flask. Increasing amounts of CTA (XD or TD) were added so that the $[\mathrm{MMA}] /[\mathrm{CTA}]$ molar ratio ranged from 0 to 0.02 . Toluene was added in the case of TD. The reaction mixture was degassed by three freeze-vacuum-thaw cycles and immersed in an oil bath at $80^{\circ} \mathrm{C}$. The polymerization was allowed to proceed at $80^{\circ} \mathrm{C}$ for a short time and then rapidly cooled down to stop the reaction at low conversion. Monomer conversions were determined by ${ }^{1} \mathrm{H}$ NMR and $M_{\mathrm{w}}$ by SEC-RI in THF. See Table S1 for details of the experiments.

Preparation of PMMA-X. XD and AIBN were dissolved in toluene in proportions according to Table 2. This solution and MMA were poured into a Schlenk flask equipped with a magnetic stirrer. The mixture was then degassed by three freeze-pump-thaw cycles and filled with argon. The flask was settled into an oil bath at $80{ }^{\circ} \mathrm{C}$ and the reaction mixture was allowed to polymerize during $6 \mathrm{~h}$. The obtained polymers were purified by precipitation in cold hexane.

Preparation of PMMA-T. MMA, TD, AIBN and MeCN were introduced in a Schlenk flask to the desired quantities. The flask was degassed by three freeze-vacuum-thaw cycles, sealed under Argon and immersed in an oil bath at $80^{\circ} \mathrm{C}$ for the desired time. The polymerization was stopped by rapid cooling. The volatiles were removed in vacuo, the residue was dissolved in the minimum amount of acetonitrile and the resulting viscous oil was filtered to remove unreacted TD. The polymer was then recovered by precipitation in cold methanol.

Preparation of PMMA-b-PVAc from PMMA-X1. PMMA-X1 (0.11 mmol), AIBN $(0.33 \mathrm{mmol})$ and VAc $(32 \mathrm{mmol})$ were dissolved in ethyl acetate $(1 \mathrm{~mL})$. The mixture was poured into a Schlenk flask and degassed by three freeze-pump-thaw cycles before being filled with argon. The flask was immersed into an oil bath at $60{ }^{\circ} \mathrm{C}$ and stirred during $6 \mathrm{~h}$. The volatiles were removed in vacuo and the polymer was recovered.

Preparation of PMMA-b-PVAc from PMMA-T. PMMA-T $(0.12$ $\mathrm{mmol})$, AIBN (0.04 mmol) and VAc (34.8 mmol) were dissolved in ethyl acetate $(1 \mathrm{~mL})$. The mixture was transferred in a Schlenk tube equipped with a septum, degassed by three freezevacuum-thaw cycles and filled with Argon. The flask was immersed in an oil bath at the desired temperature (60 or 70 ${ }^{\circ} \mathrm{C}$ ) and samples withdrawn from the polymerization mixture at different times for ${ }^{1} \mathrm{H}$ NMR and SEC analyses.

Preparation of PMMA-b-PE from PMMA-T4. An autoclave reactor $(160 \mathrm{~mL})$ was equipped with a mechanical stirring apparatus, a thermometer and a pressure sensor. A degassed solution of PMMA-T4 (9.12 $\left.\mathrm{mmol} \mathrm{L}^{-1}\right)$ and AIBN (3.04 mmol L-1) in DMC $(50 \mathrm{~mL})$ was added to the reactor preheated to $80^{\circ} \mathrm{C}$ under argon atmosphere with a mechanical stirring of $600 \mathrm{rpm}$. Immediately after the injection port was closed, ethylene gas was fed into the reactor until the targeted pressure of $80 \mathrm{bar}$ was reached. If necessary, additional ethylene gas was introduced to keep a constant pressure during the polymerization. After a predetermined period of time, the stirring was slowed down and the reactor was cooled with iced water. When the temperature inside the reactor dropped below $25{ }^{\circ} \mathrm{C}$, the remaining pressure was carefully released. The content of the reactor was collected and evaporation of the solvent gave the polymeric product. ${ }^{1} \mathrm{H}$ NMR and HT-SEC analyses were performed on the polymers without further purification.

\section{Results and discussion}

\section{Dithiocarbonate functionalized PMMA}

The Mayo method was used to determine the chain transfer constant $\left(C_{t r}\right)$ to $X D .{ }^{38}$ In this study, the weight average degree of polymerization $\left(D P_{w}\right)$ was preferred to the number average degree of polymerization $\left(D_{n}\right)$ as it is less susceptible to errors when analyzing the SEC chromatograms (e.g. baseline selection). ${ }^{39} \mathrm{~A} \mathrm{C}_{\mathrm{tr}}$ value of $0.80 \pm 0.02$ was calculated (Fig. S3), meaning that $X D$ reacts slightly more slowly than MMA during polymerization. This was confirmed by monitoring the MMA and XD conversions by ${ }^{1} \mathrm{H}$ NMR and SEC-UV at $290 \mathrm{~nm}$, respectively, for different $A I B N$ and $X D$ initial concentrations at $80^{\circ} \mathrm{C}$ (Table 1, Fig. S4). The rates of consumption of MMA and $X D$ increase with increasing initial AIBN concentration as expected for a conventional free radical polymerization. The experimental number-average molar masses $\left(M_{n, S E C}\right)$ are in relatively good agreement with theoretical values $\left(M_{n, \text { theo }}\right)$ calculated on the assumption that initiation and termination can be neglected and chain transfer to XD predominates with no RAFT activation of PMMA-X. The latter assumption of irreversible chain transfer is supported by the established low reactivity of $S$-tertiary alkyl $O$-ethyl xanthates in RAFT polymerization of MMA $\left(\mathrm{C}_{\mathrm{tr}} \sim 10^{-2}\right) \cdot{ }^{18} \mathrm{M}_{\mathrm{n} \text {,SEC }}$ determined with MALS detection and $M_{n \text {,theo }}$ match particularly well in the case of entry 3 of Table 1, for which the AIBN concentration is the lowest of the series. More generally, entries 1 to 5 of Table 1 
show that XD concentration influences $M_{\mathrm{n}}$ to a much greater extent than AIBN. Dispersities $(\Theta)$ are significantly lower than the expected value of 2.0 for a polymerization where chain transfer is the main chain breaking event. ${ }^{40}$ This may be because $\bigoplus$ is often underestimated due to weak scattering by low molar mass polymers and band broadening, both of which result in overestimation of $M_{n}{ }^{41,42}$

Table 1 Experimental details of a series of MMA polymerizations in toluene at different $X D$ and AlBN concentrations. $[\mathrm{MMA}]=4.1 \mathrm{~mol} \mathrm{~L}^{-1}, \mathrm{~T}=80^{\circ} \mathrm{C}, \mathrm{t}=6 \mathrm{~h}$.

\begin{tabular}{|c|c|c|c|c|c|c|c|c|}
\hline Entry & $\begin{array}{c}{[\mathrm{XD}]} \\
\left(\mathrm{mmol} \mathrm{L}^{-1}\right)\end{array}$ & $\begin{array}{c}{[\mathrm{AIBN}]} \\
\left(\mathrm{mmol} \mathrm{L}^{-1}\right)\end{array}$ & $\begin{array}{c}\text { Conv. } \text { MMA }^{\mathrm{a}} \\
(\%)\end{array}$ & $\begin{array}{c}\text { Conv.xD } \\
(\%)\end{array}$ & $\begin{array}{c}M_{\mathrm{n}, \text { theo }^{\mathrm{c}}} \\
\left(\mathrm{g} \mathrm{mol}^{-1}\right)\end{array}$ & $\begin{array}{c}M_{\mathrm{n}, \mathrm{SEC}}{ }^{\mathrm{d}} \\
\left(\mathrm{g} \mathrm{mol}^{-1}\right)\end{array}$ & $\begin{array}{c}M_{\mathrm{w}, \mathrm{SEC}}{ }^{\mathrm{d}} \\
\left(\mathrm{g} \mathrm{mol}^{-1}\right)\end{array}$ & $\bigoplus^{\mathrm{d}}$ \\
\hline Blank & 0 & 41 & 98 & --- & --- & 19450 & 32450 & 1.67 \\
\hline 1 & 41 & 41 & 95 & 86 & 11050 & 9400 & 14200 & 1.51 \\
\hline 2 & 41 & 20 & 91 & 77 & 11750 & 9150 & 15100 & 1.65 \\
\hline 3 & 41 & 10.2 & 79 & 68 & 11700 & 12500 & 18950 & 1.53 \\
\hline 4 & 205 & 205 & 95 & 89 & 2100 & 2650 & 3700 & 1.41 \\
\hline 5 & 205 & 102 & 93 & 72 & 2600 & 3150 & 5100 & 1.63 \\
\hline 6 & 205 & 41 & 82 & 57 & 2919 & 4500 & 5900 & 1.32 \\
\hline
\end{tabular}

${ }^{\text {a }}$ Determined by ${ }^{1} \mathrm{H} \mathrm{NMR} ;{ }^{b}$ Determined with UV detector of SEC in THF at $290 \mathrm{~nm}$ with a XD calibration curve; ${ }^{\mathrm{c}} \mathrm{M}_{\mathrm{n}, \text { theo }}=\left([\mathrm{MMA}]_{0} * \operatorname{Conv}(\mathrm{MMA})\right) /\left([\mathrm{XD}]_{0} * \mathrm{Conv}(\mathrm{XD})\right)^{*} \mathrm{MW}(\mathrm{MMA})$; d Determined by SEC-RI-MALS in THF

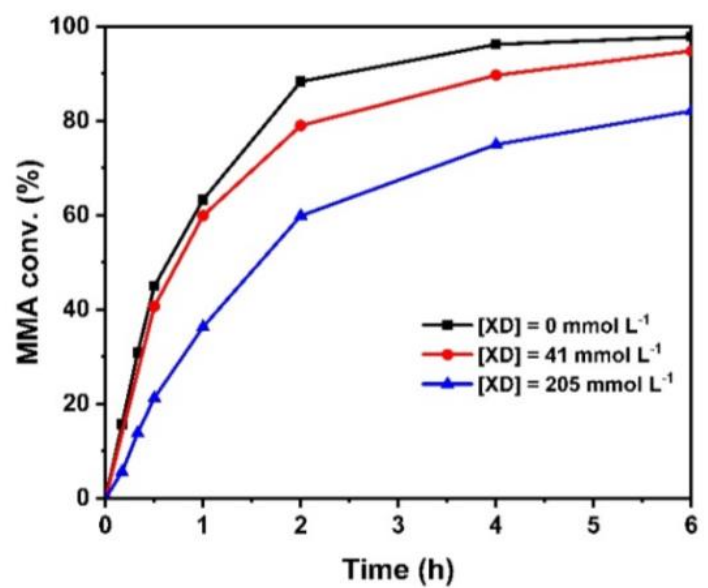

Fig. $1 \mathrm{MMA}$ conversion as a function of time for different $\mathrm{XD}$ concentrations. [AIBN] $]_{0}=$ $41 \mathrm{mmol} \mathrm{L}^{-1}$. (For more details refer to Table 1 ).

Fig. 1 shows that XD retards MMA polymerization under the reaction conditions of Table 1 . This is most likely due to slow reinitiation by the xanthyl radical. ${ }^{43}$

We studied the influence of initial AIBN and XD concentrations on the terminal xanthate functionality $f$ for four different PMMA-X (Table 2). The xanthate functionality $f$ was calculated from SEC analysis and ${ }^{1} \mathrm{H}$ NMR of precipitated PMMA. The integration of the signal at $\delta=4.55-4.77 \mathrm{ppm}$ (methylene protons $\mathrm{b}$ of the xanthate chain-end) at $\delta=3.5-3.65 \mathrm{ppm}$ (methoxy group a of MMA repeating units, Eq. 1, Fig. 2 and S5). As expected for a telomerization (i.e. a radical polymerization for which irreversible chain transfer is the main event for the formation and deactivation of polymer chains), a xanthate telechelic PMMA with $f=2.0$ was obtained after precipitation of the crude product for the lowest $[A I B N]_{0} /[X D]_{0}=1 / 10$ of the series (PMMA-X2). This important result can be explained by the efficient chain transfer to $X D$ and addition of the resulting xanthyl radical EtO- $(\mathrm{C}=\mathrm{S})-\mathrm{S} \cdot$ to MMA to create a new chain. This result has been confirmed by MALDI-TOF MS analysis (vide infra). The higher the AIBN to XD initial concentration ratio, the lower $f$ with $f=1.6$ for $[\mathrm{AIBN}]_{0} /[\mathrm{XD}]_{0} \approx 1 / 4$ (PMMA-X4) and $f \approx 1$ for $[\mathrm{AIBN}]_{0} /[\mathrm{XD}]_{0}=1$ (PMMA-X1 and $-\mathrm{X} 3$ ), attributed to an increasing contribution of AIBN-initiated chains. It is worth pointing out that only for PMMA-X1, the purification of the crude product by precipitation was accompanied by the loss of $15 \%$ of the xanthate terminal groups (Fig. S5). This was determined by comparing $f$ values calculated from Eq.1 taking into account that crude PMMA contains $\sim 25 \%$ unreacted XD (whose methylene signals overlap with those of terminal xanthate groups). This unexpected phenomenon is much more pronounced when PMMA-X samples were precipitated twice, with halved $f$ values between first and second precipitation. The instability of PMMA-X is not yet understood because in our experience, other xanthate-capped polymers (e.g. polystyrene, polyacrylates or polyvinyl acetate) do not exhibit such degradation. Consequently, the purification of PMMA-X was limited to one precipitation in cold hexane. In addition, the use of THF to dissolve the polymer between each precipitation was avoided (due to the presence of peroxides which could cause chain-end cleavage). As well, the use of the rotary evaporator and high vacuum for long periods of time were avoided and the polymer was protected from light.

$$
\boldsymbol{f}=\frac{\frac{\mathbf{1}}{\mathbf{2}}\left(\int_{4.55}^{4.77} \mathrm{CH}_{2}\right)}{\frac{\mathbf{1}}{\mathbf{3}}\left[\int_{3.50}^{\mathbf{3 . 6 5}} \mathrm{OCH}_{3}\right]} * D P_{n, S E C}
$$

Eq.1 Determination of the functionality of $\mathrm{PMMA}-\mathrm{X}$, with $\mathrm{DP}_{\mathrm{n}, \mathrm{SEC}}=$ degree of polymerization obtained from $M_{\mathrm{n}, \mathrm{SEC}}$ 
Table 2 Series of PMMA samples with different XD and AIBN concentrations and their xanthate-end functionalities; $[\mathrm{MMA}]=4.1 \mathrm{~mol} \mathrm{~L}^{-1}, \mathrm{~T}=80^{\circ} \mathrm{C}$. Solvent $=$ toluene.

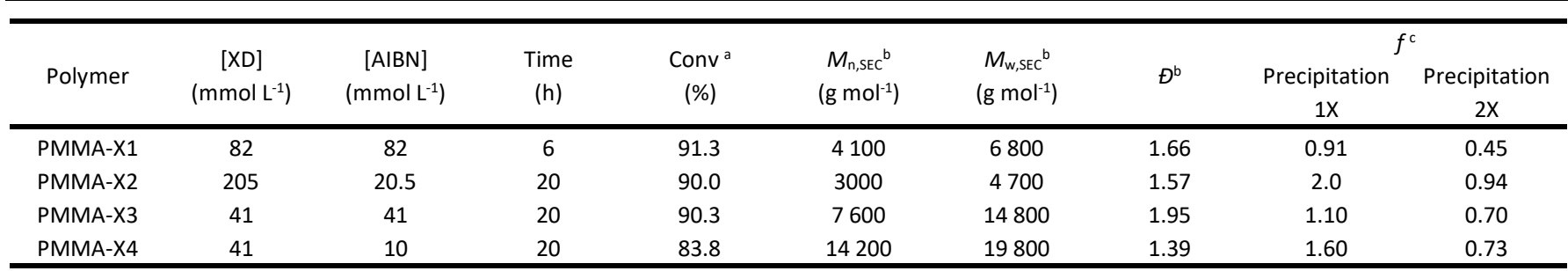

${ }^{\text {a }}$ Determined by ${ }^{1} \mathrm{H} \mathrm{NMR} ;{ }^{b}$ Determined by SEC-RI-MALS in THF; ${ }^{\mathrm{c}}$ Determined by Eq.1

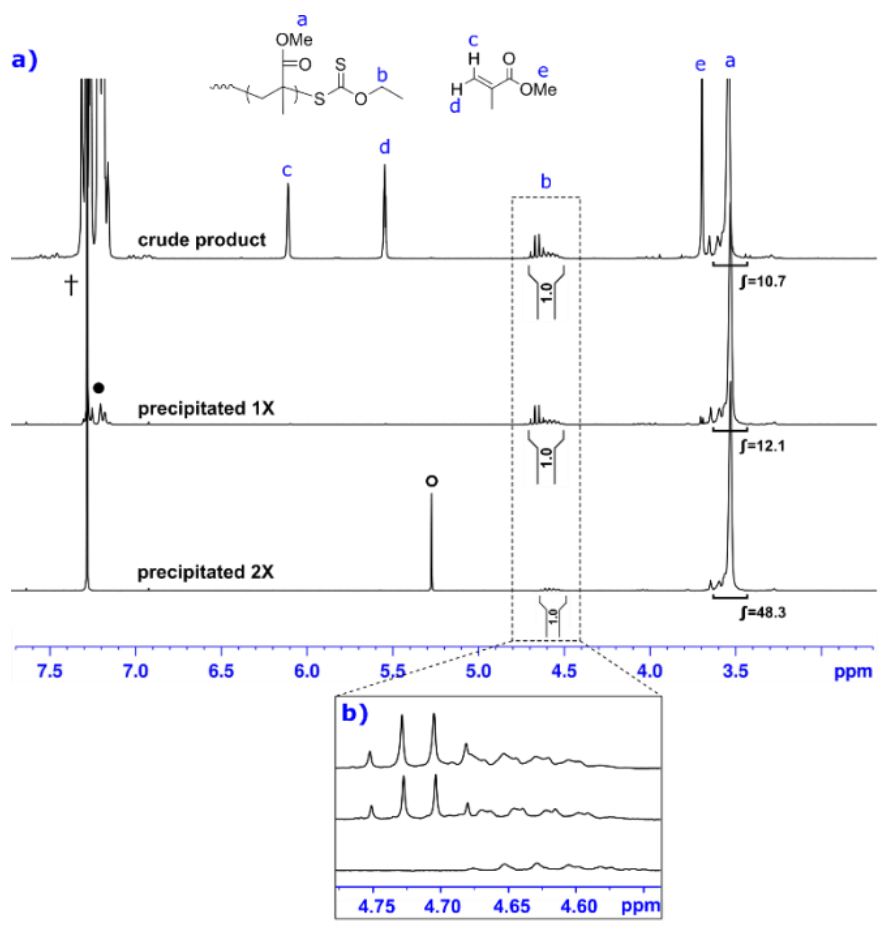

Fig. 2 a) Comparison of ${ }^{1} \mathrm{H}$ NMR spectra of PMMA-X2 $\left(M_{\mathrm{n}}=3000 \mathrm{~g} \mathrm{~mol}^{-1}\right)$ at different stages: crude product, precipitated once and precipitated twice, with their respective integration values of the xanthate chain-end (4.55-4.77 ppm) and PMMA (3.50-3.65 $\mathrm{ppm}$ ) regions. b) Zoom in the characteristic zone of the xanthate chain end. Analysis performed in $\mathrm{CDCl}_{3}$. $\left(^{\dagger}\right) \mathrm{NMR}$ solvent $\mathrm{CDCl}_{3}$. (•) residual toluene, (o) residual dichloromethane.

The MALDI-TOF spectrum of the crude low molar mass difunctional PMMA-X2 (Fig. 3a) features five main structures S1-S5. As expected, S5 (corresponding to xanthate-telechelic PMMA) has the highest intensity. Structures S3 and S4, respectively containing a single $\alpha$-xanthate group and an unsaturated or saturated $\omega$ chain-end, are present at lower intensity and presumably result from laser-induced cleavage of the terminal $\omega$-xanthate group as reported by several authors for other RAFT agent/monomer systems. ${ }^{44,45}$ As ${ }^{1} \mathrm{H}$ NMR shows the complete absence of vinylic protons signals between 5 and $6 \mathrm{ppm}$, the formation of S3 and S4 by disproportionation during polymerization can be excluded. Finally, structures containing cyanoisopropyl groups derived from AIBN (S1 and S2) are detected at low intensities. The MALDI-TOF MS spectrum of PMMA-X2 precipitated once (Fig. 3 b) indicates that the fraction of $\mathrm{S} 5$ decreased considerably, here again due to the analytical method. Populations S1-S4 are still present in the precipitated polymer. The presence of xanthate end-groups makes PMMA-X candidates of choice as macro-CTAs for RAFT polymerization of VAc. 
${ }_{[\mathrm{M}+\mathrm{Na}]^{+}=}^{\mathrm{S} 1}=1493.0$

(calc. 1492.8)

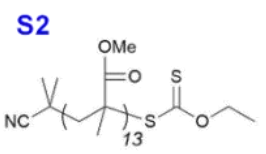

(calc. 1512.7)

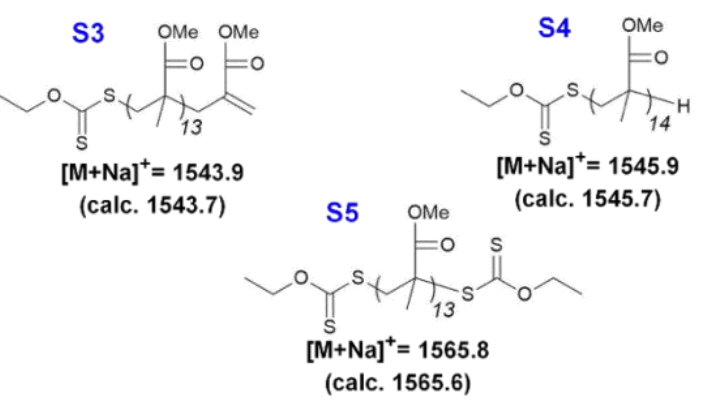

$[\mathrm{M}+\mathrm{Na}]^{+}=1512.9$
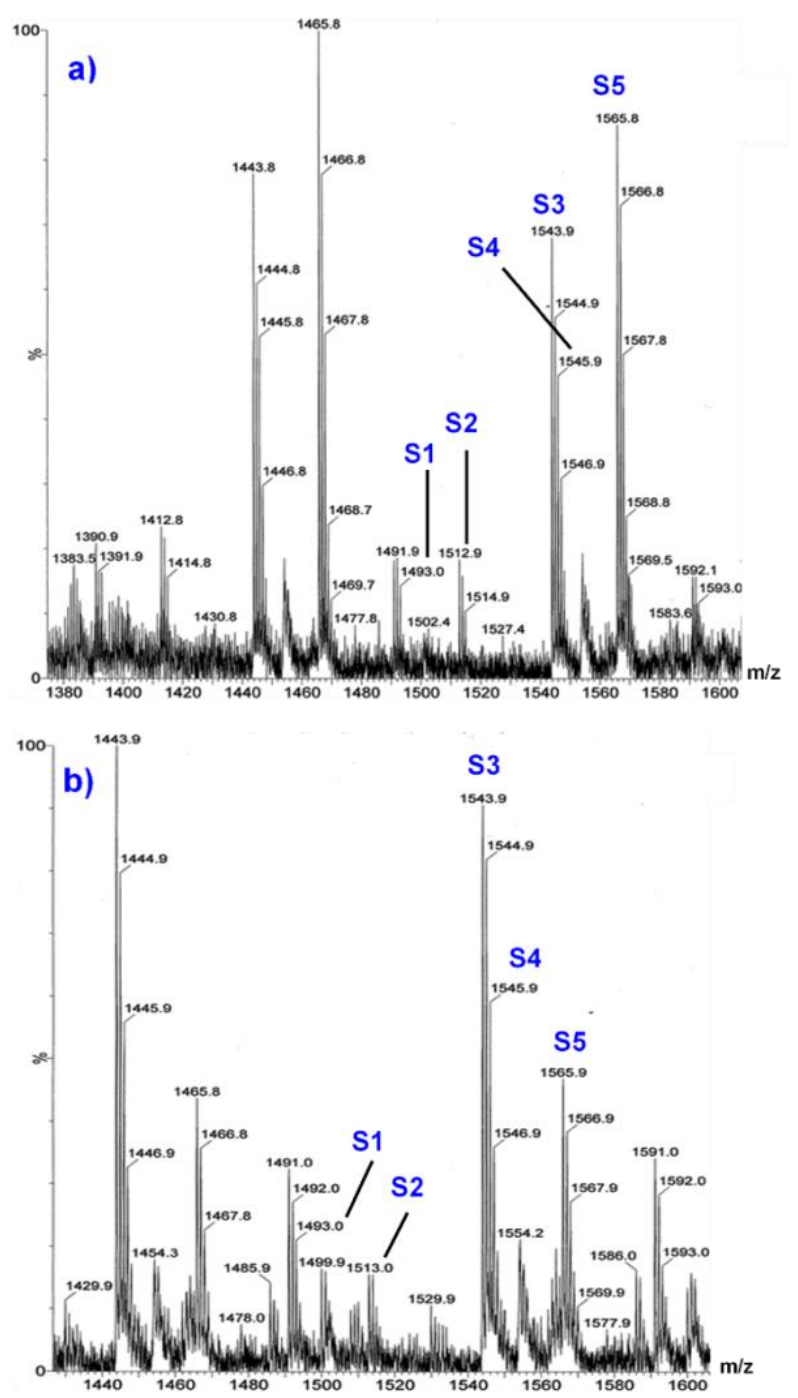

Fig. 3 MALDI-TOF MS spectra and identified species S1-S5 of PMMA-X2 $\left(M_{n}=3000 g\right.$ $\mathrm{mol}^{-1}$ ) a) crude product, b) purified by one precipitation in cold hexane.

\section{PMMA-b-PVAc block copolymer}

A PMMA-X1 sample (purified by one precipitation in cold hexane) was used as macro-CTA for the synthesis of the block copolymer with VAc by RAFT (experimental details available in
Table S2). The experimental molar masses are very close to the theoretical values. This means that despite the slight loss of functionality during the purification of PMMA-X1, the copolymerization is well controlled. It is worth pointing out that for VAc polymerization, an unusually high concentration of AIBN $\left([\mathrm{AIBN}]_{0} /[\mathrm{PMMA}-\mathrm{X}]_{0} \sim 3\right)$ was necessary to initiate the polymerization, which started after a 1 hour induction period. These initiation conditions together with a small fraction of nonfunctional PMMA $(f=0.91)$ and a non-negligible proportion of side reactions at high VAc conversions ${ }^{5}$ can explain the relatively high dispersity value of 1.8 . Fig. 4 depicts the SEC traces (RI and UV traces are shown in Fig. S6) of PMMA- $b$-PVAc compared with PMMA-X1, with the expected clean shift of the PMMA-X1 molar-mass distribution (MMD) to the higher molar mass region after chain extension. For a PMMA-X with $f=0.91$, a fraction of chains is difunctional. Even though tertiary $\omega$ xanthates are expected to show much greater activity than the primary $\alpha$-xanthates, a dual activation is likely to occur to yield $a$ fraction of PVAc- $b$-PMMA- $b$-PVAc in addition to the corresponding diblock. This hypothesis is supported by recent works of some of the co-authors on xanthate-mediated RAFT of ethylene. ${ }^{35}$ While the obtained results gave higher dispersity than what is obtained with switchable RAFT agents our proposed methodology is easier to implement as there is no need for protonation/deprotonation.

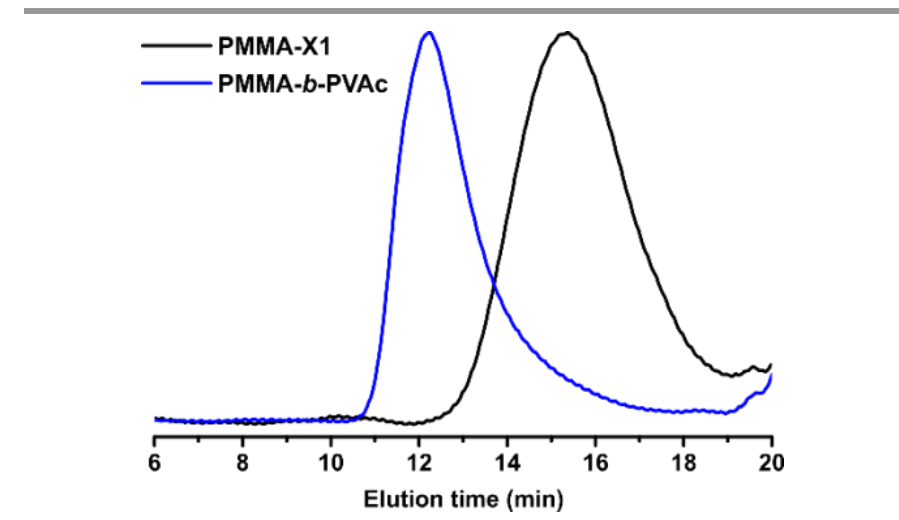

Fig. 4 SEC traces of PMMA- $b$-PVAc compared with starting macroCTA PMMA-X1.

\section{Dithiocarbamate functionalized PMMA.}

The synthesis of PMMA- $b$-PVAc block copolymers with dithiocarbamates has been reported only once, using a switchable $N$-(4-pyridinyl)- $N$-methyldithiocarbamate ${ }^{12}$ to afford a narrow PMMA- $b$-PVAc $\left(M_{\mathrm{n}}=55900 \mathrm{~g} \mathrm{~mol}^{-1}, \emptyset=1.39\right)$.

$N, N^{\prime}$-dimethyl- $N, N^{\prime}$-diphenyl thiuram disulfide (TD, Scheme 1) was investigated as an alternative to $X D$ for the synthesis of functional PMMA with a dithiocarbamate chain-end (PMMA-T, Scheme 1). The same procedure used for the determination of $\mathrm{C}_{\mathrm{tr}}$ with XD was applied, except that a minimal amount of toluene was added to the reaction mixture to ensure the complete solubility of TD at $80^{\circ} \mathrm{C}$ for all the concentrations of the disulfide used for this experiment (from $4.2 \mathrm{mmol} \mathrm{L}^{-1}$ up to $422 \mathrm{mmol} \mathrm{L}^{-1}$ ). The Mayo plot of $2 / \mathrm{DP}_{\mathrm{w}} \mathrm{vs}[\mathrm{TD}] /[\mathrm{MMA}]$ gives a straight line, the slope of which gives $C_{t r}=0.64 \pm 0.02$ (Figure S7). The chain transfer constant of toluene to MMA is $2 \times 10^{-5}$ which is negligible by comparison. ${ }^{43}$ The $C_{t r}$ value is slightly lower than 
that for $\mathrm{XD}\left(\mathrm{C}_{\mathrm{tr}}=0.80 \pm 0.02\right)$ and is very similar to values reported earlier for other thiuram disulfides. ${ }^{46}$ TD is thus consumed more slowly than XD during the polymerization. Both TD and XD have chain transfer constants that are fairly close to the ideal value of 1 . This is advantageous as the ratio of CTA to monomer will remain approximately constant throughout the polymerization.

For the preparation of polymer for chain extension, acetonitrile was preferred to toluene as polymerization solvent. TD is almost completely insoluble in cold acetonitrile (solubility $<5 \mathrm{mg} \mathrm{mL}^{-1}$ ), whereas PMMA is readily soluble (solubility $>100 \mathrm{mg} \mathrm{mL}^{-1}$ ). Thus, unreacted TD could be efficiently removed from the
PMMA by filtration of the crude product dispersed in a minimal amount of cold acetonitrile. The subsequent precipitation of the polymer in a large excess of cold methanol allowed the isolation of PMMA free of unreacted TD. Using this method, we synthesized four different PMMA-T (experimental conditions summarized in Table 3). Their corresponding molar mass features $\left(M_{n}, \oplus\right)$ are listed in the same table. Functionality $f$ was calculated with Eq. 2 and the integral values of the aromatic protons a-c of the dithiocarbamate chain-end $(\delta=7.35-7.55$ $(3 \mathrm{H})$ and $\delta=7.25-7.15 \mathrm{ppm}(2 \mathrm{H}))$ and of the methoxy protons $\mathrm{f}$ of the PMMA main chain ( $\delta=3.50-3.65 \mathrm{ppm})$ obtained by ${ }^{1} \mathrm{H}$ NMR (Fig. 5 in the case of PMMA-T3).

Table 3 Experimental details for the synthesis of PMMA-T

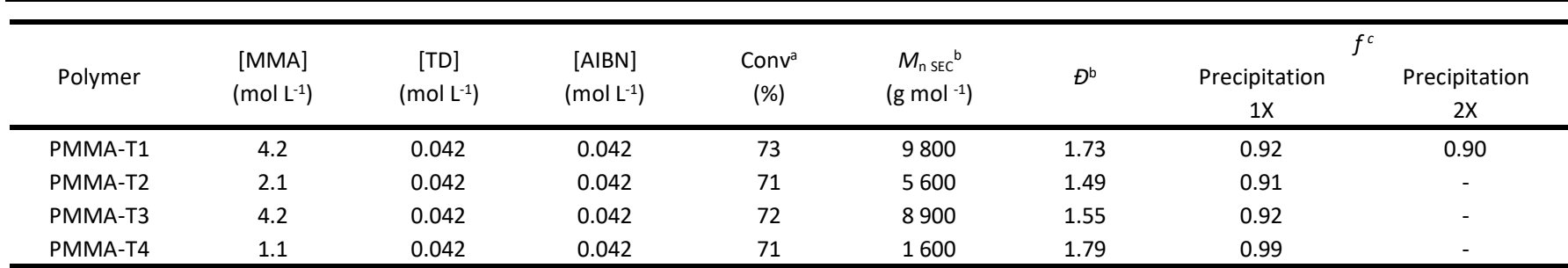

Polymerizations carried out in acetonitrile at $80{ }^{\circ} \mathrm{C}$ during $6 \mathrm{~h} .{ }^{a}$ Conversion of MMA determined by ${ }^{1} \mathrm{H}$ NMR, ${ }^{\mathrm{b}}$ Determined by SEC-RI in THF, conventional PMMA calibration, ${ }^{\mathrm{C}}$ determined by Eq. 2 .

$$
f=\frac{D P_{n, S E C}}{D P_{n, N M R}}=\frac{\frac{\mathbf{1}}{\mathbf{5}}\left[\left(\int_{7.35}^{7.55} C_{6} H_{5}\right)+\left(\int_{7.15}^{7.25} C_{6} H_{5}\right)\right]}{\frac{1}{3}\left[\int_{3.50}^{3.65} \text { OCH }_{3}\right]} * D P_{n, S E C}
$$

Eq. 2 Determination of the functionality of PMMA-T, with $D P_{\mathrm{n}, \mathrm{SEC}}=$ degree of polymerization obtained from $M_{\mathrm{n}, \mathrm{SEC}}$

By changing the [MMA]:[TD] ratios (100, 50 and 25) different $M_{\mathrm{n}}$ values of PMMA-T can be achieved $\left(M_{n}=8900,5600\right.$ and 1600 $\mathrm{g} \mathrm{mol}^{-1}$, respectively) with limited impact on the dispersity $(\theta=$ 1.55, 1.49 and 1.79, respectively). PMMA-T1 was precipitated twice with no apparent loss of functionality (compared to PMMA-X for which functionality was halved after two precipitations). This indicates a better stability of the $\omega$ chainend when $\mathrm{Z}=\mathrm{N}(\mathrm{Me}) \mathrm{Ph}$.
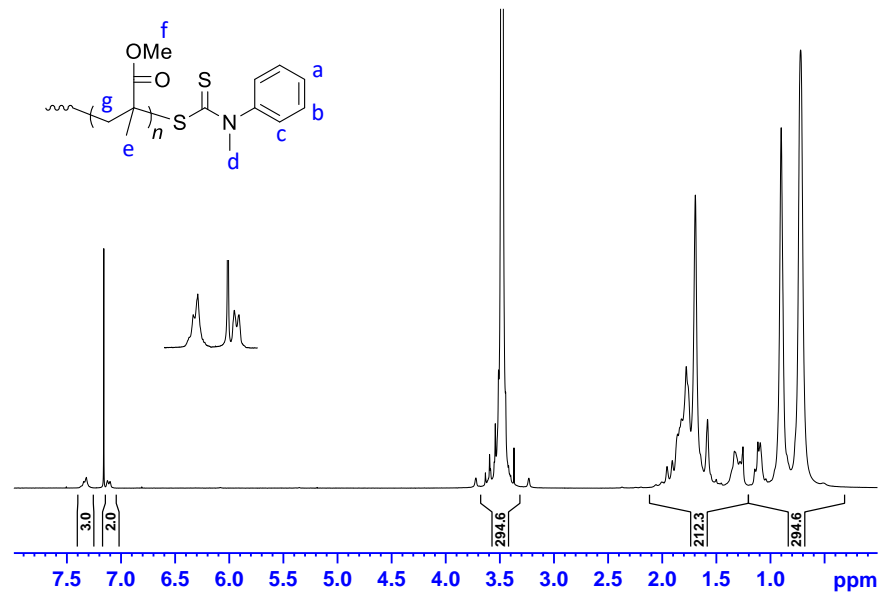

Fig. $5^{1} \mathrm{H}$ NMR spectrum of PMMA-T3 after isolation $\left(M_{\mathrm{n}, \mathrm{SEC}}=8900 \mathrm{~g} \mathrm{~mol}^{-1}\right)$.
The presence of $\sim 0.9$ dithiocarbamate end-group per chain on average is confirmed by the protons in the aromatic region that integrate for three $(\delta=7.35-7.55)$ and two protons $(\delta=7.25$ 7.15). The characteristic multiplet of ten aromatic protons $(\delta=$ 7.45-7.65, see Fig. S8) of TD is not observed.

MALDI-TOF MS analysis performed on PMMA-T2 (Fig. 6) confirms monofunctionalization. The main species observed corresponds to a PMMA chain featuring a double bond at the $\omega$ chain-end (T3). ${ }^{1} \mathrm{H}$ NMR analysis does not show vinylic protons between 5 and $6 \mathrm{ppm}$ while the aromatic protons from the dithiocarbamate moiety are undoubtedly assigned (Fig. 5 for PMMA-T3 and Fig. S8 for PMMA-T2). Consequently, T3 and T4 correspond to the expected monofunctionalized PMMA chains carrying a dithiocarbamate $\omega$ chain-end that underwent degradation in the spectrometer during ionization. Indeed, a small quantity of PMMA still bearing the dithiocarbamate chainend (T1) is detected as well. MALDI-TOF MS shows no sign of telechelic PMMA, either before $\omega$ chain-end loss due to ionization (T2) or after chain-end loss (T5). Our results confirm the conclusions of Otsu et al. who reported that TD is a very poor thermal iniferter compared to tetraalkyl counterparts. ${ }^{28,47}$ 


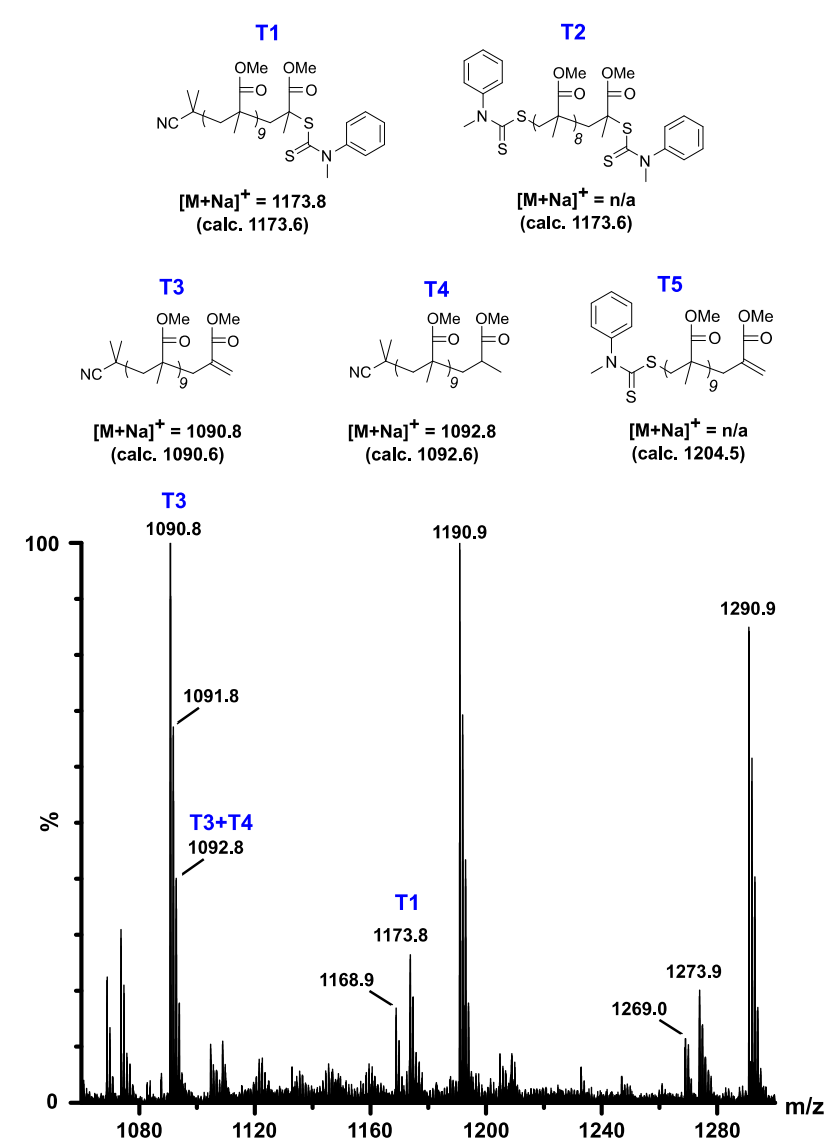

Fig. 6 MALDI-TOF MS spectrum of PMMA-T2 (5600 $\mathrm{g} \mathrm{mol}^{-1}$ ) and identification of corresponding species after precipitation in methanol.

SEC analysis confirms the presence of a thiocarbonylthio moiety on the entire MMDs of PMMA-T2 and PMMA-T3 as the UV absorbance at $290 \mathrm{~nm}$ (corresponding to the maximum absorbance of the $\mathrm{C}=\mathrm{S}$ double bond) is consistent with the refractive index (RI) trace (Fig. 7). UV and RI traces are slightly offset relative to each other because the UV detector is sensitive only to the RAFT agent and hence gives the number distribution of the polymer, while the RI detector is sensitive to the mass concentration of polymer in solution and hence gives the weight distribution. Thus low molar mass chains that may form as a result of the peculiar mechanism of initiation/primary recombination give a stronger signal in the UV trace.
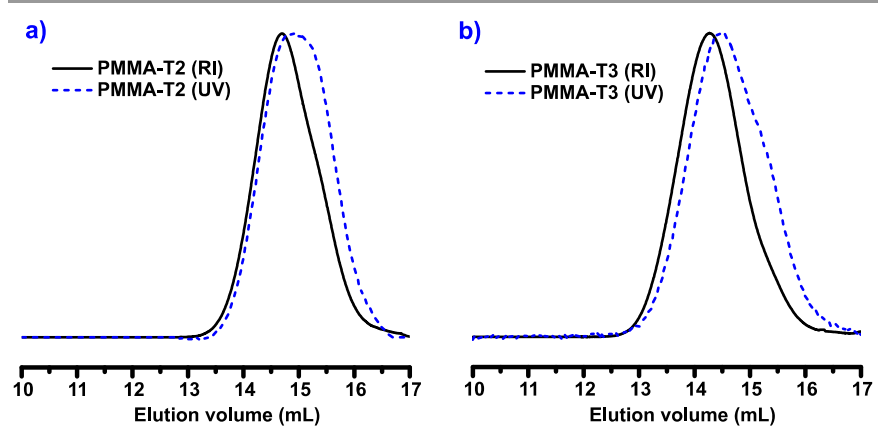

Fig. 7 SEC-THF traces (- RI) and (--- UV) of PMMA-T2 (a) and PMMA-T3 (b).

PMMA-b-PVAc block copolymers
The resulting PMMA carrying a dithiocarbamate chain-end could be chain extended with VAc. Although the control on the PMMA segment could not compete with that obtained using a switchable CTA, similar block copolymers can be obtained in a more convenient way. PMMA-T2 and PMMA-T3 were used as macro-CTA for the synthesis of PMMA- $b$-PVAc copolymers by RAFT at $60^{\circ} \mathrm{C}$ and $70^{\circ} \mathrm{C}$ respectively. A strong induction period was observed at $60^{\circ} \mathrm{C}$ with no polymerization taking place over the first 6 hours (Fig. S10) and a conversion of only $20 \%$ was reached after 20 hours (Fig.S9 and Fig.S10). This induction period could be reduced to less than 1 hour by performing the chain extension at $70{ }^{\circ} \mathrm{C}$ and $90 \%$ of conversion could be reached after 6 hours of polymerization (Fig. S10). The presence of an induction period may be due to slow addition of the PMMA macroradical to VAc. The results of the chain extension with PMMA-T3 at $70^{\circ} \mathrm{C}$ are presented in Fig. 8. A clean shift of the MMD towards higher values is observed (Fig. 8a) and the $M_{n}$ determined by both ${ }^{1} \mathrm{H}$ NMR and SEC-RI are close to theoretical values while $\Theta$ decreases from 1.55 to 1.43 as the polymerization proceeds (Fig. 8b). This decrease in dispersity is consistent with expectations for a well-controlled polymer. ${ }^{48}$

a)

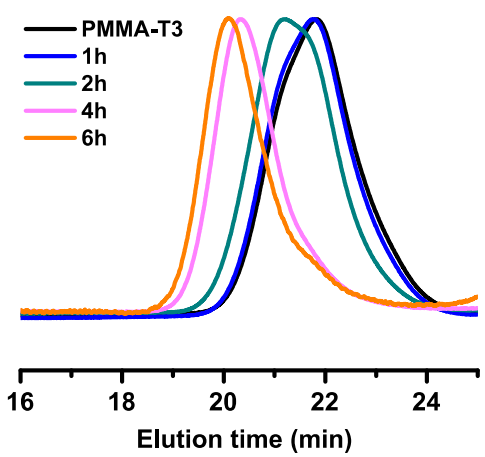

b)

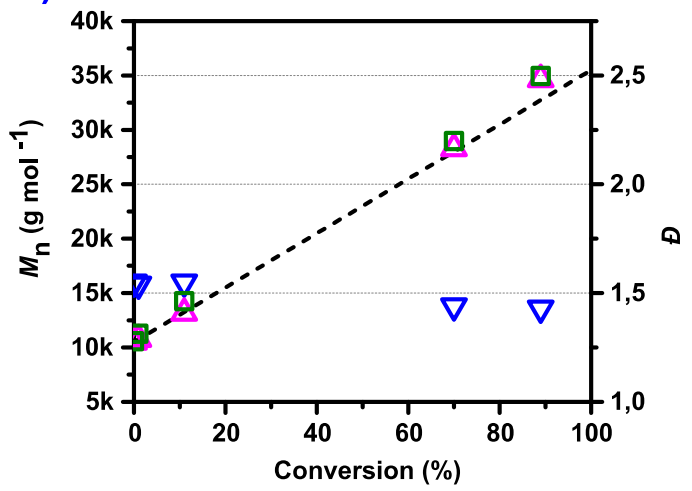

Fig. 8 a) SEC-RI traces of the chain extension of PMMA-T3 with VAc. b) evolution of the corresponding $M_{n, N M R}\left(\triangle\right.$, determined by integration of PVAc $-\left(\mathrm{CH}_{2}-\mathrm{CH}(\mathrm{OAC})\right)$ - signal relative to $-\left(\mathrm{CH}_{2}-\mathrm{C}\left(\mathrm{CH}_{3}\right)\left(\mathrm{C}(\mathrm{O}) O C \mathrm{H}_{3}\right)\right)$ - signal), $M_{\mathrm{n}, \mathrm{SEC}}(\square$, calibration PMMA standards) and $\theta(\boldsymbol{\nabla})$ values of PMMA- $b$-PVAc made with PMMA-T3 at $70{ }^{\circ} \mathrm{C}$. The dotted line corresponds to the theoretical $M_{\mathrm{n}}$ values calculated for $f=1$.

The linear increase of $M_{\mathrm{n}}$ values with conversion of VAc and their good agreement with the theoretical values (calculated for $f=1$ ) confirms that the majority of PMMA-T bears one dithiocarbamate chain-end. The minor tailing observed at 
elution time $=22$ minutes after 6 hours of polymerization most likely originates from the small fraction of non-functionalized PMMA. The growth of the PVAc block proceeds in a controlled fashion.

\section{PMMA-b-PE copolymers}

We recently demonstrated that RDRP based on reversible degenerative transfer such as tellurium mediated radical polymerization (TeRP) ${ }^{49}$ or RAFT, ${ }^{35}$ could be used to control the polymerization of ethylene. In the case of RAFT, aromatic dithiocarbamates are particularly efficient to obtain welldefined PE chains. ${ }^{36}$ PMMA-T4 (Table 3) $\left(M_{\mathrm{n}}=1600 \mathrm{~g} \mathrm{~mol}^{-1}\right.$, high chain-end fidelity of 0.99 ) was used for chain extension with ethylene at $80{ }^{\circ} \mathrm{C}$ and 80 bar using DMC as solvent. The chain-extension was performed in an autoclave reactor and, because of the difficulty of sampling under pressure, each polymerization times $(2 \mathrm{~h}, 3 \mathrm{~h}, 4 \mathrm{~h}, 5 \mathrm{~h})$ represent an independent experiment. The results of the chain extension are presented in Fig. 9. A clean shift of the MMD towards higher elution times is observed (Fig. 9a) via HT-SEC. Noteworthy, the conditions used for HT-SEC $\left(150^{\circ} \mathrm{C}, 1,2,4\right.$-trichlorobenzene) are adapted for the analysis of PE but not PMMA and result in the apparent $M_{n}$ and $Ð$ of PMMA-T4 being substantially different for those obtained by SEC-THF $\left(M_{\mathrm{n}}=450 \mathrm{~g} \mathrm{~mol}^{-1}\right.$ and $\theta=3.38$ via HT-SEC (PS calibration) versus $M_{\mathrm{n}}=1600 \mathrm{~g} \mathrm{~mol}^{-1}$ and $D=1.79$ via SEC-THF (PMMA calibration)). The behavior of the targeted PMMA- $b-\mathrm{PE}$ during HT-SEC analyses and the corresponding determined molar mass characteristics are thus to be taken with care. Nonetheless, the molar masses of the block copolymers obtained after chain extension increase with the conversion of ethylene, expressed as the yield of ethylene (Fig. 9b). a)

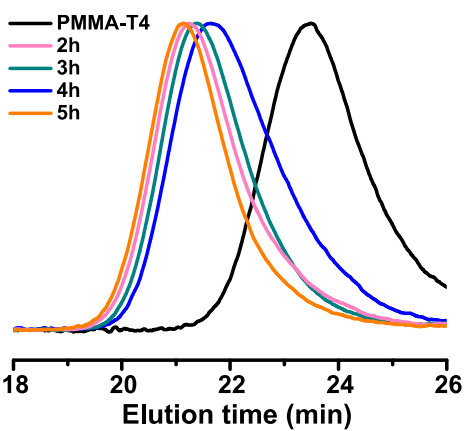

b)

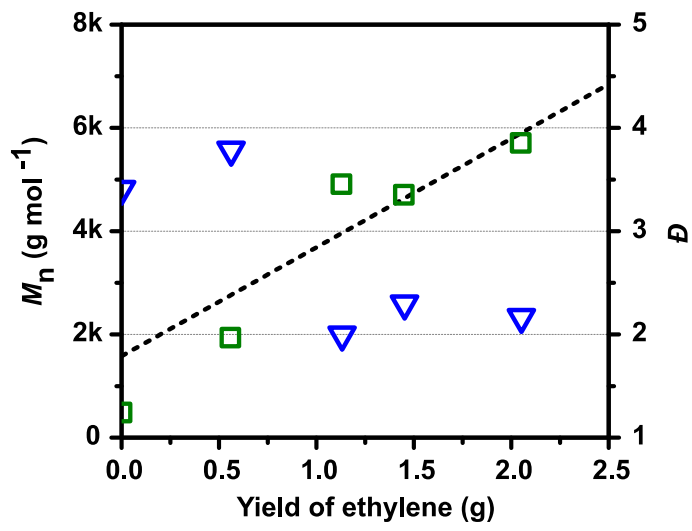

Fig. 9 a) HT-SEC-RI traces of the chain extension of PMMA-T4 with ethylene. b) evolution of the corresponding $M_{n, H T-S E C}(\square$, calibration PS standards) and $D(\nabla)$ values of PMMA$b$-PE made with PMMA-T4 at $80^{\circ} \mathrm{C}$. The dotted line corresponds to the theoretical $M_{\mathrm{n}}$ values calculated for $f=1$.

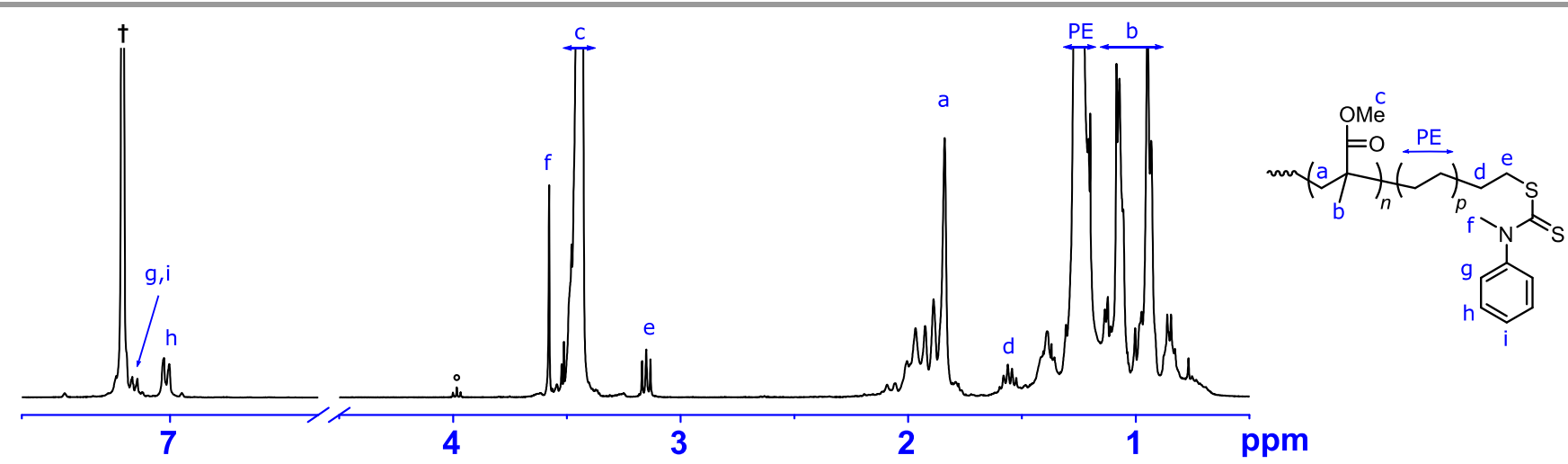

Fig $10^{1} \mathrm{H}$ NMR spectrum of PMMA- $b$-PE after 5 hours of chain extension of PMMA-T4 with ethylene at $80^{\circ} \mathrm{C}$ and 80 bar. + : NMR residual solvent benzene; ${ }^{\circ}$ : transfer to DMC 35,36

A ${ }^{1} \mathrm{H}$ NMR spectrum of the polymer obtained after 5 hours of chain extension of PMMA-T4 with ethylene is presented in Fig. 10. The characteristic signals of $P E$ and $P M M A$ protons can be observed (methoxy protons c of PMMA at $3.45 \mathrm{ppm}$, methylene protons of PMMA between 1.7 and $2.1 \mathrm{ppm}$, methylene protons of $\mathrm{PE}$ and methyl protons of PMMA between 0.9 and $1.5 \mathrm{ppm}$ ). In addition, the presence of $d$ and e of the $\mathrm{PE}-\mathrm{CH}_{2}-\mathrm{CH}_{2}-\mathrm{SC}(\mathrm{S}) \mathrm{Z}$ species indicate that ethylene units have been inserted between the PMMA segment and the dithiocarbamate chain end. Aromatic protons $g, l, h$ and methyl protons $f$ of the dithiocarbamate chain ends are visible between 7 and $7.5 \mathrm{ppm}$ and at $3.58 \mathrm{ppm}$, respectively. HT-SEC, together with ${ }^{1} \mathrm{H} N M R$ analyses, confirm the successful chain extension of PMMA-T4 and the synthesis of the desired PMMA- $b$-PE copolymers, for the first time achieved using a free radical process and for the first time using a RDRP technique to produce the PE block.

\section{Conclusions}


In summary, the conventional free radical polymerization of MMA in the presence of xanthogen or thiuram disulfide transfer agents afforded PMMA functionalized with the corresponding xanthate or dithiocarbamate chain-end. XD afforded both monofunctionalized and telechelic PMMA depending on relative $A I B N$ and $X D$ concentrations. Xanthate-terminated PMMA exhibited relatively low stability with gradual decrease of chain-end functionality during the purification process. However, degradation could be limited by precipitating the polymer only once. This instability may be due to the lability of the $\omega$-O-ethyl xanthogenate group when bonded to a MMA unit. Under the presently used polymerization conditions, TD afforded exclusively monofunctionalized PMMA that maintains high chain-end fidelity during the handling process. Xanthate and dithiocarbamate functionalized PMMA with a functionality greater than $90 \%$ were successfully used for the synthesis of well-defined block copolymers with VAc by a RAFT process. As xanthogen and thiuram disulfides are commonly used industrial vulcanization accelerators for rubber manufacturing, they may serve as more accessible alternatives to switchable CTAs for the synthesis of MAM-LAM block copolymers containing polyethylene segments.

\section{Conflicts of interest}

There are no conflicts to declare.

\section{Acknowledgements}

B.F.M. acknowledges the financial support from Consejo Nacional de Ciencia y Tecnologia (CONACYT, Mexico) to pursue her Ph.D. C.B., F.D. and V.M. thank ANR (Agence Nationale de la Recherche) for funding the project PolarOBC ANR-15-CE070015.

\section{Notes and references}

1
A. D. Jenkins, R. G. Jones and G. Moad, Pure Appl. Chem., 2009, 82, 483-491.

W. A. Braunecker and K. Matyjaszewski, Prog. Polym. Sci., 2007, 32, 93-146.

M. Destarac, Macromol. React. Eng., 2010, 4, 165-179.

M. Destarac, Polym. Chem., 2018, 9, 4947-4967.

S. Harrisson, X. Liu, J.-N. Ollagnier, O. Coutelier, J.-D. Marty and M. Destarac, Polymers, 2014, 6, 1437-1488.

K. Matyjaszewski, Macromolecules, 2012, 45, 4015-4039.

G. Moad, E. Rizzardo and S. H. Thang, Aust. J. Chem., 2012, 65, 985.

R. Nicolaÿ, Y. Kwak and K. Matyjaszewski, Chem. Commun., 2008, 5336.

Y. Y. Tong, Y. Q. Dong, F. S. Du and Z. C. Li, Macromolecules, 2008, 41, 7339-7346.

S. Yamago, Chem. Rev., 2009, 109, 5051-5068.

B. Ray, M. Kotani and S. Yamago, Macromolecules, 2006, 39, 5259-5265.

M. Benaglia, J. Chiefari, Y. K. Chong, G. Moad, E. Rizzardo

and S. H. Thang, J. Am. Chem. Soc., 2009, 131, 6914-6915.

13

14

15

16

17

18

19

20

21
G. Moad, M. Benaglia, M. Chen, J. Chiefari, Y. K. Chong, D. J. Keddie, E. Rizzardo and S. H. Thang, ACS Symp. Ser., 2011, 1066, 81-102.

D. J. Keddie, C. Guerrero-Sanchez, G. Moad, E. Rizzardo and S. H. Thang, Macromolecules, 2011, 44, 6738-6745.

G. Moad, D. Keddie, C. Guerrero-Sanchez, E. Rizzardo and S. H. Thang, Macromol. Symp., 2015, 350, 34-42.

J. Gardiner, I. Martinez-Botella, J. Tsanaktsidis and G. Moad, Polym. Chem., 2016, 7, 481-492.

E. Girard, T. Tassaing, J. D. Marty and M. Destarac, Polym. Chem., 2011, 2, 2222-2230.

M. Destarac, D. Matioszek, X. Vila, J. Ruchmann-Sternchuss and S. Z. Zard, ACS Symp. Ser., 2018, 1284, 291-305.

L. A. Dayter, K. A. Murphy and D. A. Shipp, Aust. J. Chem., 2013, 66, 1564-1569.

S. Sistach, M. Beija, V. Rahal, A. Brûlet, J. D. Marty, M. Destarac and C. Mingotaud, Chem. Mater., 2010, 22, 37123724.

A. Guinaudeau, O. Coutelier, A. Sandeau, S. Mazières, H. D. Nguyen Thi, V. Le Drogo, D. J. Wilson and M. Destarac, Macromolecules, 2014, 47, 41-50.

K. Hakobyan, T. Gegenhuber, C. S. P. McErlean and M. Müllner, Angew. Chemie - Int. Ed., 2019, 58, 1828-1832. J. Gardiner, I. Martinez-Botella, T. M. Kohl, J. Krstina, G. Moad, J. H. Tyrell, M. L. Coote and J. Tsanaktsidis, Polym. Int., 2017, 66, 1438-1447.

J. Zeng, J. Zhu, X. Pan, Z. Zhang, N. Zhou, Z. Cheng, W. Zhang and X. Zhu, Polym. Chem., 2013, 4, 3453-3457. W. Chen, Z. Shen, X. Huang and J. Huang, Macromol. Rapid Commun., 1997, 18, 197-205.

S. Maiez-Tribut, J. P. Pascault, E. R. Soulé, J. Borrajo and R. J. J. Williams, Macromolecules, 2007, 40, 1268-1273.

T. Otsu and K. Nayatani, Die Makromol. Chemie, 1958, 27, 149-156.

T. Otsu, K. Nayatani, I. Muto and M. Imai, J. Chem. Soc., 1958, 61, 142-148.

M. Niwa, T. Matsumoto and H. Izumi, J. Macromol. Sci. Part A - Chem., 1987, 24, 567-585.

T. Otsu and M. Yoshida, Polym. Bull., 1982, 7, 197-203. M. Niwa, Y. Sako and M. Shimizu, J. Macromol. Sci. Part A Chem., 1987, 24, 1315-1332.

S. A. Haque, J. Macromol. Sci. Part A, 1994, 31, 827-833. J-M. Catala, WO 03/010773 A2, 2003.

C. Zhang, L. Li, H. Cong and S. Zheng, J. Polym. Sci. Part A Polym. Chem., 2014, 52, 952-962.

C. Dommanget, F. D'Agosto and V. Monteil, Angew. Chem. Int. Ed., 2014, 53, 6683-6686.

A. Wolpers, C. Bergerbit, B. Ebeling, F. D'Agosto and V. Monteil, Angew. Chem. Int. Ed., 2019, 58, 14295.

L. Shi, T. M. Chapman and E. J. Beckman, Macromolecules, 2003, 36, 2563-2567.

F. R. Mayo, J. Am. Chem. Soc., 1943, 65, 2324-2329.

J. P. A. Heuts, T. P. Davis and G. T. Russell, Macromolecules, 1999, 32, 6019-6030.

G. Odian, Princ. Polym., 2004, 3, 198-349.

L. Seiler, J. Loiseau, F. Leising, P. Boustingorry, S. Harrisson 
and M. Destarac, Polym. Chem., 2017, 8, 3825-3832. Y. Guillaneuf and P. Castignolles, J. Polym. Sci. Part A Polym. Chem., 2008, 46, 897-911.

43 G. Moad and D. H. Solomon, in The Chemistry of Radical Polymerization (Second Edition), eds. G. Moad and D. H. Solomon, Elsevier Science Ltd, Amsterdam, Second Edi., 2005, pp. 279-331. X. Jiang, P. J. Schoenmakers, J. L. J. van Dongen, X. Lou, V. Lima and J. Brokken-Zijp, Anal. Chem., 2003, 75, 55175524.

45 L. Charles, Mass Spectrom. Rev., 2014, 33, 523-543.

46 S. A. Haque and G. Clouet, Macromol. Chem. Phys., 1994, 195, 315-327.

47 T. Otsu, J. Polym. Sci., 1956, 21, 559-561.

48 S. Harrisson, Polym. Chem., 2018, 9, 1366-1370.

49 Y. Nakamura, B. Ebeling, A. Wolpers, V. Monteil, F. D'Agosto and S. Yamago, Angew. Chem. Int. Ed., 2018, 57, 305-309. 